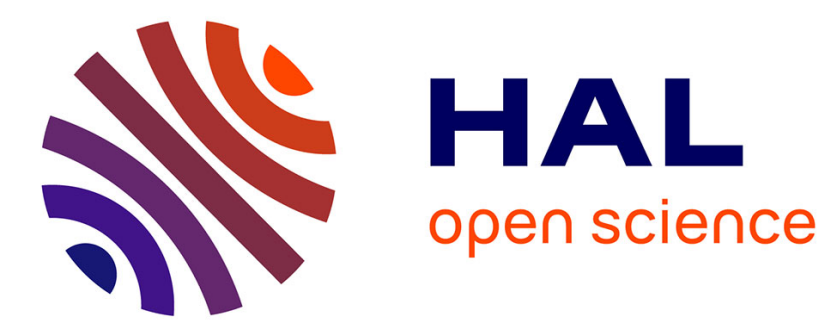

\title{
EQUICONTINUITY AND LI-YORKE PAIRS OF DENDRITE MAPS
}

Ghassen Askri

\section{To cite this version:}

Ghassen Askri. EQUICONTINUITY AND LI-YORKE PAIRS OF DENDRITE MAPS. 2019. hal02006196

\section{HAL Id: hal-02006196 \\ https://hal.science/hal-02006196}

Preprint submitted on 4 Feb 2019

HAL is a multi-disciplinary open access archive for the deposit and dissemination of scientific research documents, whether they are published or not. The documents may come from teaching and research institutions in France or abroad, or from public or private research centers.
L'archive ouverte pluridisciplinaire HAL, est destinée au dépôt et à la diffusion de documents scientifiques de niveau recherche, publiés ou non, émanant des établissements d'enseignement et de recherche français ou étrangers, des laboratoires publics ou privés. 


\title{
EQUICONTINUITY AND LI-YORKE PAIRS OF DENDRITE MAPS
}

\author{
GHASSEN ASKRI
}

\begin{abstract}
In this paper, relationships between equicontinuity of a dendrite map $f$ on $\Lambda(f)$, absence of Li-Yorke pairs, collection of minimal sets and regularly recurrent points are investigated.
\end{abstract}

\section{Introduction}

Let $(X, f)$ be a dynamical system i.e. $X$ is a compact metric space with metric $d$ and $f: X \rightarrow X$ is a continuous map. Denote by $\mathbb{N}$ the set $\{1,2,3, \ldots\}$ and $\mathbb{Z}_{+}=\mathbb{N} \cup\{0\}$. For any $n \in \mathbb{N}, f^{n}=f^{n-1} \circ f$ where $f^{0}$ is the identity map.

1.1. Topological dynamics. Let $x \in X$. The orbit of $x$ (under $f$ ) is $\operatorname{Orb}_{f}(x)=\left\{f^{n}(x) ; n \in \mathbb{Z}_{+}\right\}$and the $\omega$-limit set of $x$ is $\omega_{f}(x)=\{y \in X$ : $\left.\lim \inf _{n \rightarrow+\infty} d\left(f^{n}(x), y\right)=0\right\}$. The point $x$ is called

- fixed if $f(x)=x$ and $N$-periodic (or periodic with period $N$ ) if $f^{N}(x)=x$ and $f^{i}(x) \neq x$ for $0<i<N$,

- regularly recurrent if for any open set $U$ containing $x, \operatorname{Orb}_{f^{N}}(x) \subset U$ for some $N \in \mathbb{N}$,

- almost periodic if for any open set $U$ containing $x$, there is $N \in \mathbb{N}$ such that for all $i \in \mathbb{Z}_{+},\left\{f^{i}(x), f^{i+1}(x), \ldots, f^{i+N}(x)\right\} \cap U \neq \emptyset$,

- recurrent if $x \in \omega_{f}(x)$.

We denote by $\Lambda(f)$ the union of all $\omega$-limit sets and Fix $(f), P(f), R R(f)$, $A P(f)$ and $R(f)$ the sets of fixed points, periodic points, regularly recurrent points, almost periodic points and recurrent points, respectively. Recall that always we have

$$
P(f) \subset R R(f) \subset A P(f) \subset R(f) \subset \Lambda(f) .
$$

A nonempty subset of $X$ is called minimal if it is closed, $f$-invariant and has no proper subset with these properties. The map $f$ is called minimal if $X$ itself is minimal. Notice that $M$ is minimal if and only if any point in it is almost periodic. Let $A$ be a subset of $X$. Then $A$ is called $N$-periodic (or periodic with period $N$ ) if $A, f(A), \ldots, f^{n-1}(A)$ are pairwise disjoint and $f^{N}(A)=A$. The orbit of $A$ (under $f$ ) is the set $\operatorname{Orb}_{f}(A)=\cup_{n \in \mathbb{Z}_{+}} f^{n}(A)$.

2010 Mathematics Subject Classification. 37B05, 37B45, 37E99.

Key words and phrases. dendrite map, minimal set, equicontinuous map, Li-Yorke pair, recurrent point. 
A point $y \in X$ is called an $\alpha$-limit point of $x$ under $f$ if and only if there is a strictly increasing sequence of positive integers $\left(k_{n}\right)_{n \geq 0}$ and a sequence of points $\left(y_{n}\right)_{n \geq 0}$ in $X$ such that

- $f^{k_{n}}\left(y_{n}\right)=x$ for all $n \geq 0$, and

- $\lim _{n \rightarrow+\infty} y_{n}=y$.

The set $\alpha_{f}(x)$ of all $\alpha$-limit points of $x$ under $f$ is called the $\alpha$-limit set.

1.2. General topology. For $A \subset B \subset X, \operatorname{Int}(A, B)$ mean the interior of $A$ relatively to $B$. For any subset $A$ of $X, A^{\prime}$ is the set of accumulation points of $A$.

\subsection{Li-Yorke pairs and entropy.}

- A pair $(x, y) \in X^{2}$ is called

- proximal if $\liminf _{n \rightarrow+\infty} d\left(f^{n}(x), f^{n}(y)\right)=0$,

- asymptotic if $\lim _{\sup } \operatorname{su}_{n \rightarrow+\infty} d\left(f^{n}(x), f^{n}(y)\right)=0$,

- distal they are not proximal,

- a $L i$-Yorke pair if it is proximal but not asymptotic.

The map $f$ is called distal if $(x, y) \in X^{2}$ is proximal then $x=y$.

- Let $n \in \mathbb{N}$ and $\varepsilon>0$. A subset $E$ of $X$ is called $(n, \varepsilon)$-separated if for any distinct points $x, y \in E$, we have $\max _{0<i<n} d\left(f^{i}(x), f^{i}(y)\right)>$ $\varepsilon$. Denote by $\operatorname{Sep}(n, f, \varepsilon)$ the maximal possible cardinality of an $(n, f, \varepsilon)$-separated set in $X$. The topological entropy of $f$ is defined by

$$
h(f)=\lim _{\varepsilon \rightarrow 0} \limsup _{n \rightarrow+\infty} \frac{\log \operatorname{Sep}(n, f, \varepsilon)}{n} .
$$

It was proved by Blanchard et al [4] that any dynamical system with positive topological entropy is Li-Yorke chaotic i.e. it has an uncountable set for which each proper pair is a Li-Yorke pair.

1.4. Equicontinuity. $f$ is called equicontinuous (or stable in the sense of Lyapunov) at $x \in X$ if for any $\varepsilon>0$ there is $\delta>0$ such that $d\left(f^{n}(x), f^{n}(y)\right)<$ $\varepsilon$ for all $n \geq 0$ provided that $d(x, y)<\delta$. The map $f$ is equicontinuous if it is equicontinuous at any point of $X$ or equivalently for any $\varepsilon>0$ there is $\delta>0$ such that $d\left(f^{n}(u), f^{n}(v)\right)<\varepsilon$ for all $n \geq 0$ provided that $d(u, v)<\delta$. Notice that $f$ is equicontinuous at $x$ if and only if $f^{k}$ is so at $f^{i}(x)$ for any positive integers $k$ and $i$.

1.5. Hyperspace. We denote by $2^{X}$ the hyperspace of all nonempty closed subsets of $\mathrm{X}$. The Hausdorff metric $d_{H}$ is defined as follows: Let $A, B \in 2^{X}$,

$$
d_{H}(A, B)=\max \left\{\sup _{x \in A} d(x, B), \sup _{y \in B} d(y, A)\right\}
$$

where $d(x, A)=\inf _{y \in A} d(x, y)$. The space $2^{X}$ endowed with $d_{H}$ is a compact metric space. 
1.6. Dendrites. A dendrite is a locally connected compact connected metric space containing no simple closed curve. Let $X$ be a dendrite. A point $x \in X$ is called endpoint (resp. branch point) if $X \backslash\{x\}$ is connected (resp. has at least three connected components). We denote by $\operatorname{End}(X)$ and $B(X)$ the sets of endpoints and branch points of $X$, respectively. A triod is a dendrite with unique branch point and finitely many endpoints. Any two distinct points $a$ and $b$ in $X$ can be joined by a unique arc, we denote it by $[a, b]$. We denote by $[a, b)=[a, b] \backslash\{b\}$ and $(a, b)=[a, b] \backslash\{a, b\}$. The convex hull of a nonempty subset $A$ of $X$ is the connected subset $[A]=\cup_{x \in A}[a, x]$ for any $a \in A$. An arc $I$ of $X$ is called free if $I \cap B(X)=\emptyset$. Let $Y$ be a subdendrite of $X$ and $x \in Y$. The order of $x$ relatively to $Y$, denoted by $\operatorname{ord}_{Y}(x)$, is the number of connected components of $Y \backslash\{x\}$. For any $x \in X$, let $r_{Y}(x)$ be the unique point in $Y$ belonging to the arc joining $x$ and $z$ for any $z \in Y$. The map $r_{Y}: X \rightarrow Y$ is called the first point map.

1.7. Motivation and Main result. Li-Yorke chaotic dynamical systems has been extensively studied in the literature during the last forty years . In such a system various kind of $\omega$-limit sets and recurrent points can occur. It is interesting to study the behavior of points of systems without Li-Yorke pairs. In [9], [5] and [6], Smital and his co-authors investigated nonchaotic interval maps.

Theorem 1.1. ([6], [5]) Let $f: I \rightarrow I$ be an interval map. Then the following assertions are equivalent:

(1) $f$ has no Li-Yorke pairs,

(2) $f_{\mid \Lambda(f)}$ is equicontinuous,

(3) $\Lambda(f)=R R(f)$.

In this paper we generalise the previous theorem on dendrites. The main result of the paper is the following.

Theorem 1.2. Let $(X, f)$ be a dynamical system. The relations between the properties:

(1) $f$ has no Li-Yorke pairs,

(2) $f_{\mid \Lambda(f)}$ is equicontinous,

(3) $R(f)=R R(f)$,

(4) the collection of minimal sets is closed in $2^{X}$,

(5) $\Lambda(f)=A P(f)$.

are described by the scheme below where the thick (resp. thin, dashed) arrow means that the implication is true for general dynamical systems(resp. true for dendrites $X$ with $\operatorname{End}(X)$ countable and closed, false). 


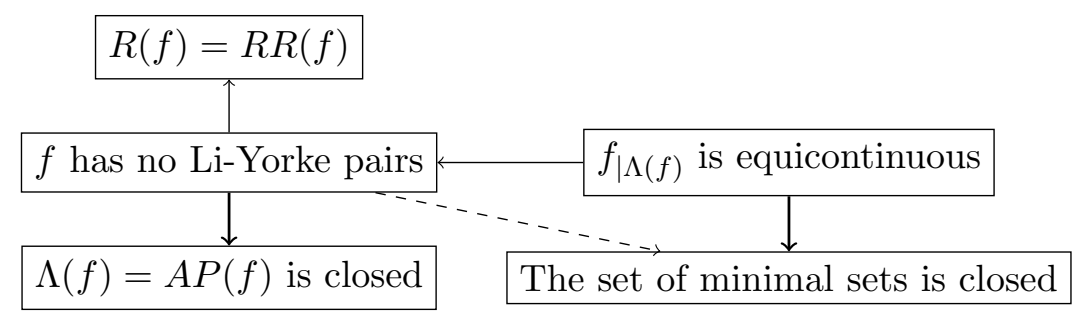

\section{Auxilary results}

Definition 2.1. Let $f: X \rightarrow X$ be a dendrite map and let $I$ and $J$ be two arcs of $X$ which are either disjoint or intersect only in their endpoint. If $f^{n}(I) \cap f^{m}(J) \supset I \cup J$ for some $n, m \in \mathbb{N}$ then we say that $I, J$ form an arc horseshoe for $f$.

Lemma 2.2. ([7], Theorem 2 ) Let $f$ be a continuous map of a dendrite. If there is an integer $n$ such that $f^{n}$ has an arc horseshoe then $h(f)>0$.

Lemma 2.3. ([3], Lemma 1$)$ Let $(X, f)$ be a dynamical system, $f$ be onto and $x \in X$. Then $\alpha_{f}(x)$ is non-empty, closed and $f$-invariant.

Lemma 2.4. [8] Let $X$ be a compact Hausdorff space and let $f: X \rightarrow X$ be a minimal map. If $U$ is a nonempty open subset of $X$ then there is a positive integer $r$ such that $X=\cup_{i=0}^{r} f^{i}(U)$.

The following result is a folklore.

Lemma 2.5. Let $(X, f)$ be a dynamical system. If $f$ is equicontinuous then it is distal. In particular, $f$ has no Li-Yorke pairs.

The following Theorem is due to Auslander and Ellis.

Theorem 2.6. Let $(X, f)$ be a dynamical system. Then for any $x \in X$ there is some almost periodic point $y \in \operatorname{Orb}_{f}(x)$ such that $(x, y)$ is proximal.

Remark. In the previous theorem, $y$ can be choosen in $\omega_{f}(x)$.

Lemma 2.7. Let $(X, f)$ be a dynamical system without Li-Yorke pairs. Then any $\omega$-limit set is minimal. In other words, $\Lambda(f)=A P(f)$.

Proof. Let $L=\omega_{f}(x)$ be an $\omega$-limit set of $f$. By Auslander-Ellis's theorem, there is a minimal set $M \subset L$ and a point $y \in M$ such that $(x, y)$ is proximal. Since $f$ has no Li-Yorke pairs then $(x, y)$ is asymptotic. It follows that $L=M$.

Lemma 2.8. Let $(X, f)$ be a dynamical system. If $f_{\mid \Lambda(f)}$ is equicontinuous then the collection of all minimal sets of $f$ is closed in $\left(2^{X}, d_{H}\right)$.

Proof. Let $\left(M_{n}\right)_{n \geq 1}$ be a sequence of minimal sets converging in $\left(2^{X}, d_{H}\right)$ to $M$. Then $M$ is a closed $f$-invariant set. Suppose that $M$ is not minimal. Then there is a proper minimal subset $F$ of $M$. Let $z \in M \backslash F, \varepsilon=d_{H}(z, F)$ and $x_{0} \in F$. By equicontinuity of $f_{\mid \Lambda(f)}$, there is $0<\delta<\frac{\varepsilon}{2}$ such that for 
all $x \in \Lambda(f)$ and all $n \in \mathbb{Z}_{+}, d\left(f^{n}\left(x_{0}\right), f^{n}(x)\right)<\frac{\varepsilon}{2}$ provided that $d\left(x, x_{0}\right)<$ $\delta$. Let $p>0$ such that $d_{H}\left(M, M_{p}\right)<\delta$. There is $x \in M_{p}$ such that $d\left(x, x_{0}\right)<\delta$. As $M_{p}$ is minimal, there is $k>0$ such that $d\left(f^{k}(x), z\right)<\frac{\varepsilon}{2}$. Then $d\left(f^{k}\left(x_{0}\right), z\right) \leq d\left(f^{k}\left(x_{0}\right), f^{k}(x)\right)+d\left(f^{k}(x), z\right)<\varepsilon$. So $d_{H}(z, F)<\varepsilon$. Absurd.

Lemma 2.9. Let $(X, f)$ be a dynamical system such that $f_{\mid \Lambda(f)}$ is equicontinuous. Then $\Lambda(f)=A P(f)$.

Proof. It is clear that $A P(f) \subset \Lambda(f)$. Suppose that there is an $\omega$-limit set $L=\omega_{f}(x)$ and $y \in L$ such that $y \notin A P(f)$. Then there is a minimal set $M \subset L$ and $z \in M$ such that $(y, z)$ is proximal. Since $f_{\mid \Lambda(f)}$ is equicontinuous then by Lemma $2.5(y, z)$ is asymptotic. On the other hand, by Lemma $2.3 \alpha_{f_{\mid L}}(y)$ is a nonempty, closed and $f$-invariant subset of $L$.

Claim. $M \subset \alpha_{f_{\mid L}}(y)$.

Proof. Suppose that $M \nsubseteq \alpha_{f_{\mid L}}(y)$. Then by minimality of $M, M \cap \alpha_{f_{\mid L}}(y)=$ $\emptyset$. Let $\varepsilon_{0}=d_{H}\left(M, \alpha_{f_{\mid L}}(y)\right)$ and let $w \in \alpha_{f_{\mid L}}(y)$. Let $\delta>0$. There is $t \in L$ such that $d(t, w)<\delta$ and $f^{n}(t)=y$ for some $n \geq 1$. So there is $N \geq 1 \mathrm{such}$ that $d\left(f^{k}(t), f^{k}(w)\right)>\frac{\varepsilon_{0}}{2}$ for all $k \geq N$. Thus $f_{\mid L}$ is not equicontinuous. Absurd. This finishes the proof of the Claim.

There is a sequence $\left(y_{n}\right)_{n \geq 1}$ in $L$ converging to $z$ and a nondecreasing sequence of integers $\left(k_{n}\right)_{n \geq 1}$ such that $f^{k_{n}}\left(y_{n}\right)=y$ for all $n \geq 1$. For any $\alpha>0$ and any $N \geq 1$, there is $n \geq N$ such that $d\left(y_{n}, z\right)<\alpha$, but $d\left(f^{k_{n}}\left(y_{n}\right), f^{k_{n}}(z)\right)=d\left(y, f^{k_{n}}(z)\right)>\frac{d(y, M)}{2}$. It follows that $f_{\mid L}$ is not equicontinuous at $z$. Absurd. Thus $\Lambda(f) \subset A P(f)$. Hence $\Lambda(f)=A P(f)$.

From Lemmas 2.8 and 2.9 we can deduce the following

Corollary 2.10. Let $(X, f)$ be a dynamical system such that $f_{\mid \Lambda(f)}$ is equicontinuous. Then $\Lambda(f)$ is closed.

Lemma 2.11. Let $(X, f)$ be a dynamical system such that $f_{\mid \Lambda(f)}$ is equicontinuous. Then $h(f)=0$.

Proof. Since $\Lambda(f)$ is closed, then $\overline{R(f)} \subset \Lambda(f)$. Hence by $[2] h(f)=$ $h\left(f_{\mid \overline{R(f)}}\right)=h\left(f_{\mid \Lambda(f)}\right)$. As $f_{\mid \Lambda(f)}$ is equicontinuous, we get $h(f)=0$.

\section{Splitting minimal sets of maps on dendrites $X$ with $\operatorname{Card}(\operatorname{End}(X))<c$}

Definition 3.1. Let $X$ be a dendrite. An arc $[x, y]$ in $X$ is called a tracing arc starting from $x$ if Fix $(f) \cap[x, y)=\emptyset$ and for any $z \in[x, y),(z, f(z)) \cap$ $[x, y] \neq \emptyset$. Moreover, if $f(y)=y$ then $[x, y]$ is called a complete tracing arc.

Lemma 3.2. ([11], Proposition 2.9) Let $f: X \rightarrow X$ be a dendrite map. Then, for any given $x \in X \backslash F i x(f)$, there exists a unique complete $f$-tracing arc starting from $x$.

The proof of the following Lemma is inspired from [1]. 
Lemma 3.3. Let $f: X \rightarrow X$ be a zero topological entropy dendrite map with $\operatorname{Card}(\operatorname{End}(X))<c$ and let $M$ be a nondegenerate (finite or infinite) minimal set. Then there is a connected subset $C$ of $X$ and an integer $k \geq 2$ satisfying the following properties:

(i) $C \cap M$ has nonempty interior in $M$,

(ii) $f^{k}(C) \subset C$,

(iii) $M \subset \cup_{i=0}^{k-1} f^{i}(C)$,

(iv) $C, f(C), \ldots, f^{k-1}(C)$ are pairwise disjoint.

Proof. Let $Y=[M]$. We distinguish two cases:

Case 1: $Y \cap F i x(f) \neq \emptyset$. Let $a \in Y$ be a fixed point. Denote by $A=\cup_{n \in \mathbb{N}} f^{-n}(a)$ and $A_{Y}=A \cap Y$. Notice that $\operatorname{ord}_{Y}(a)<\infty$ (It's clear if $M$ is finite. Suppose that $M$ is infinite and $\operatorname{ord}_{Y}(a)=$ $\infty$. Then there are infinitely many pairwise disjoint connected subsets $\left(C_{i}\right)_{i \geq 1}$ of $Y \backslash\{a\}$ such that $C_{i} \cap M \neq \emptyset$ for all $i \geq 1$. So $\lim _{i \rightarrow+\infty} \operatorname{diam}\left(C_{i}\right)=0$. Since $a \in \overline{C_{i}}$ for all $i \geq 1$, we get $a \in M$. Absurd).

Claim 1. $\left[A_{Y}\right] \cap M=\emptyset$.

Proof. Suppose the contrarily. Then there is $b \in Y$ and $y \in M$ such that $y \in(a, b)$ and $f^{n}(b)=a$ for some $n \geq 1$. Let $I=[a, y]$ and $J=[y, b]$. Since $M$ is closed, there is $z \in M$ such that $b \in(y, z)$. So there are integers $p, q>0$ such that $f^{p}(I) \cap f^{q}(J) \supset I \cup J$. Hence $I, J$ is an arc horseshoe. By Lemma 2.2, $h(f)>0$. Absurd. This finishes the proof of Claim 1.

Proof of (i) We have $M=\cup_{e \in \operatorname{End}(X)}[a, e] \cap M$. Since $\operatorname{Card}(\operatorname{End}(X))<c$, then by the well known theorem of Baire, there is $e_{0} \in \operatorname{End}(X)$ such that $\operatorname{Int}\left(\left[a, e_{0}\right] \cap M, M\right)$ is nonempty. Let $M_{0}=M \cap$ $\left[a, e_{0}\right]$. By Claim $1,\left[M_{0}\right] \cap A_{\infty}=\emptyset$. So let $C$ be the connected component of $X \backslash A_{\infty}$ containing $M_{0}$. Hence $\operatorname{Int}(M \cap C, M) \neq \emptyset$.

Proof of (ii) Let $x \in M_{0}$, there is $k>0$ such that $f^{k}(x) \in M_{0}$. So $f^{k}(C) \cap$ $C \neq \emptyset$. As $f^{k}(C) \cap A_{\infty}=\emptyset$, we get $f^{k}(C) \subset C$.

We can assume that $k$ is the smallest integer with this property.

Proof of (iii) Since $\operatorname{Int}\left(M_{0}, M\right) \neq \emptyset$, then by [8], we have $\cup_{i=0}^{N} f^{i}\left(M_{0}\right)=M$ for some $N \geq k$. As $M_{0} \subset C$, we get $\cup_{i=0}^{N} f^{i}\left(M_{0}\right) \subset \cup_{i=0}^{N} f^{i}(C)$. So using (ii), $M \subset \cup_{i=0}^{k-1} f^{i}(C)$.

Proof of (iv) Suppose on the contrary that $f^{i}(C) \cap f^{j}(C) \neq \emptyset$ for some $0 \leq$ $i<j \leq k-1$. Let $s=k-j$. Then $f^{s+i}(C) \cap f^{s+j}(C) \neq \emptyset$. Since $f^{s+j}(C)=f^{k}(C) \subset C, f^{s+i}(C) \cap C \neq \emptyset$. As $0<s+i<k$, this contradicts the minimality of $k$.

The integer $k$ is greater than 1 since $\operatorname{ord}_{Y}(a)>1$ and $M \subset$ $\cup_{i=0}^{k-1} f^{i}(C)$. 
Case 2: $Y \cap F i x(f)=\emptyset$. Let $x \in M$. Then by Lemma 3.2 there is a complete tracing arc with endpoints $x$ and $a$ where $a \in$ Fix $(f)$. By Lemma 5.5 of [1], for any $y \in Y,[y, a]$ is a complete tracing arc starting from $y$. Let $b=r_{Y}(a)$ and $B=\cup_{n \geq 0} f^{-n}(b)$. Notice that $f(b) \notin Y, b \notin M$ and $k:=\operatorname{ord}_{Y}(b)<\infty$.

Claim 2. $[B \cap Y] \cap M=\emptyset$.

Proof. Suppose the contrary. Then there are $b^{\prime} \in Y$ and $x \in M$ such that $x \in\left(b, b^{\prime}\right)$ and $f^{n}\left(b^{\prime}\right)=b$ for some $n \in \mathbb{N}$. Let $I=[b, x]$, $J=\left[x, b^{\prime}\right]$ and $y \in M$ such that $\left[x, b^{\prime}\right] \subset(b, y)$. Since $b \in[x, f(b)]$, then $f(I) \supset[b, f(x)]$. More generally, we have $f^{k}(I) \supset\left[b, f^{k}(x)\right]$ for all $k \in \mathbb{N}$. Since $y \in \omega_{f}(x)=M$, there is $N \geq n$ such that $f^{N}(I) \supset I \cup J$. On the other hand, $f^{n}(J) \supset\left[b, f^{n}(x)\right]$. So $f^{N}(J) \supset I \cup J$. Hence $I, J$ is an arc horseshoe for $f$. Absurd.

As in Case 1, there is a connected subset $C$ of $X \backslash B$ satisfying properties $(i)-(i v)$.

This ends the proof of the Lemma.

Proposition 3.4. Let $f: X \rightarrow X$ be a zero topological entropy dendrite map with $\operatorname{Card}(\operatorname{End}(X))<c$ and let $M$ be a nondegenerate minimal set. If $M$ is finite then set $\mathcal{I}=\{1,2, \ldots, p\}$. If $M$ is infinite then set $\mathcal{I}=\mathbb{N}$. Then there is a sequence of subdendrites $\left(D_{n}\right)_{n \in \mathcal{I}}$ of $X$ and a sequence of nondecreasing integers $\left(k_{n}\right)_{n \geq 1}$ satisfying the following properties:

(P.1) for any $n \in \mathcal{I}, D_{n}$ is periodic of period $k_{n}$ and $k_{n} \mid k_{n+1}$ whenever $n+1 \in \mathcal{I}$,

(P.2) for any $i, j \in\left\{0,1, \ldots, k_{n}-1\right\}(i \neq j), f^{i}\left(D_{n}\right)$ and $f^{j}\left(D_{n}\right)$ are either disjoint or intersect only in one common point,

(P.3) for any $n \geq 1, \cup_{i=0}^{q_{n}-1} f^{i k_{n}}\left(D_{n+1}\right) \subset \operatorname{Orb} b_{f}\left(D_{n}\right)$ where $q_{n}=\frac{k_{n+1}}{k_{n}}$,

(P.4) $M \subset \cap_{n \geq 1} \operatorname{Orb}_{f}\left(D_{n}\right)$,

Proof. Let $C$ be a connected subset of $X$ satisfying properties $(i)-(i v)$ of Lemma 3.3. For any $0 \leq i \leq k_{1}-1$, we have $f\left(M_{i}\right)=M_{i+1 \bmod k_{1}}$ where $M_{i}=f^{i}(C) \cap M$. Let $D_{1}=\cap_{i=0}^{+\infty} f^{k_{1} i}(\bar{C})$. Then

- $D_{1}$ is a subdendrite of $X$ and $f^{k_{1}}\left(D_{1}\right) \subset D_{1}$,

- for any $0 \leq i<j<k_{1}, f^{i}\left(D_{1}\right)$ and $f^{j}\left(D_{1}\right)$ are either disjoint or intersect in one common point.

- for any $0 \leq i<k_{1}, M_{i} \subset f^{i}\left(D_{1}\right)$. So $M \subset \operatorname{Orb}_{f}\left(D_{1}\right)$.

Now, $f_{\mid D_{1}}^{k_{1}}: D_{1} \rightarrow D_{1}$ is a dendrite map of zero topological entropy and $\operatorname{Card}\left(\operatorname{End}\left(D_{1}\right)\right)<c$. Since $M_{0}$ is a minimal set of $f_{\mid D_{1}}^{k_{1}}$, there is a periodic subdendrite $D_{2} \subset D_{1}$ under $f_{\mid D_{1}}^{k_{1}}$ of period $q_{1} \geq 2$ such that $M_{0} \subset \cup_{j=0}^{q_{1}-1} f^{k_{1} j}\left(D_{2}\right), f^{k_{1} i}\left(D_{2}\right)$ and $f^{k_{1} j}\left(D_{2}\right)\left(0 \leq i \neq j<q_{1}\right)$ are either disjoint or intersect only on one common point. So $D_{2}$ is a periodic subdendrite of period $k_{2}=k_{1} q_{1}$ such that $M \subset \operatorname{Orb}_{f}\left(D_{2}\right)$ and $\cup_{i=0}^{q_{1}-1} f^{i k_{1}}\left(D_{2}\right) \subset \operatorname{Orb}\left(D_{1}\right)$. We continue this process to construct the sequence $\left(D_{n}\right)_{n \geq 1}$ satisfying properties $\left(P_{1}\right)-\left(P_{4}\right)$. 
Lemma 3.5. Let $X$ be a dendrite with $\operatorname{End}(X)$ countable and closed and let $\left(D_{n}\right)_{n \in \mathbb{N}}$ be a sequence of subdendrites of $X$ satisfying $(P .1),(P .2)$ and (P.3). Then there is an integer $N \geq 1$ such that $\forall n \geq N, f^{i}\left(D_{n}\right)$ is a free arc for some $0 \leq i<k_{n}$.

Proof. Suppose that for any integer $n$ and any $0 \leq i<k_{n}, f^{i}\left(D_{n}\right) \cap$ $\operatorname{End}(X) \neq \emptyset$. Then for any $n \geq 1$, the subsets $E_{n, i}=f^{i}\left(D_{n}\right) \cap \operatorname{End}(X)$ $\left(0 \leq i<k_{n}\right)$ are pairwise disjoint and closed. Let $\tau=\left(i_{0}, i_{1}, i_{2}, \ldots\right) \in$ $\prod_{n=0}^{+\infty} \mathbb{Z} / q_{n} \mathbb{Z}$ where $q_{0}=k_{0}=1$. By the property $\left(P_{3}\right)$, the subset $\cap_{n=1}^{+\infty} f^{\sum_{j=0}^{n-1} i_{j} k_{j}}\left(D_{n}\right)$ is nonempty, closed and intersect $\operatorname{End}(X)$. Furthermore, $E_{\tau} \cap E_{\tau^{\prime}}=\emptyset$ whenever $\tau \neq \tau^{\prime}$. Pick $x_{\tau} \in E_{\tau} \cap \operatorname{End}(X)$. Then the map $\tau \in \prod_{n=0}^{+\infty} \mathbb{Z} / q_{n} \mathbb{Z} \mapsto$ $x_{\tau} \in \operatorname{End}(X)$ is injective. This implies that $\operatorname{End}(X)$ is uncountable. Absurd. So there is $N \in \mathbb{N}$ such that $f^{i}\left(D_{N}\right) \cap \operatorname{End}(X)=\emptyset$ for some $0 \leq i<k_{N}$. Since $\operatorname{End}(X)$ is closed, $f^{i}\left(D_{N}\right) \cap B(X)$ is finite. By taking $k_{n}$ sufficiently large, we have $f^{j}\left(D_{n}\right) \cap B(X)=\emptyset$ for some $n \geq N$ and $0 \leq j<k_{n}$. It follows that $f^{j}\left(D_{n}\right)$ is a free arc.

\section{Li-Yorke pairs and equicontinuity of a dendrite map $f$ on $\Lambda(f)$}

Lemma 4.1. Let $X$ be a dendrite with $\operatorname{End}(X)$ is countable and closed and let $f: X \rightarrow X$ be a continuous map. If $f_{\mid \Lambda(f)}$ is equicontinuous then $f$ has no Li-Yorke pairs.

Proof. Suppose on the contrary that $(x, y)$ is a Li-Yorke pair of $f$. By Lemma $2.9 \omega_{f}(x) \cup \omega_{f}(y) \subset A P(f)$. We can assume that $\omega_{f}(x)$ is infinite. By [10] $\omega_{f}(x) \nsubseteq P(f)$. So there is an infinite minimal set $M \subset \omega_{f}(x)$. By Lemma $2.11 h(f)=0$. So by Proposition 3.4 and Lemma 3.5 there is a periodic free arc $I$ of $X$ of period $p$ such that $M \subset \operatorname{Orb}_{f}(I)$. Set $g=f^{p}$. Then $M_{0}:=M \cap I$ is an infinite minimal set of $g$. There is $n \geq 0$ such that $f^{n}(x) \in I$. Since $\left(f^{n}(x), f^{n}(y)\right)$ is a Li-Yorke pair of $g$, there is a nondecreasing sequence of integers $\left(n_{i}\right)_{i \geq 1}$ such that $\left(g^{n_{i}}\left(f^{n}(x)\right)\right)_{i \geq 1}$ and $\left(g^{n_{i}}\left(f^{n}(y)\right)\right)_{i \geq 1}$ converges to some $z \in I$. If $g^{m}(f(y)) \in I$ for some $m \geq 1$ then $\left(f^{n}(x), f^{n}(y)\right)$ is a Li-Yorke pair for $g_{\mid I}$. Otherwise, $z \in \partial(I)$. Hence $g^{2}(z)=z$. So $\left(f^{n}(x), z\right) \in I^{2}$ is also a Li-Yorke pair for $g_{\mid I}$. In all cases, the interval map $g_{\mid I}: I \rightarrow I$ has a Li-Yorke pair. By [6] $g_{\mid I}$ is not equicontinuous on $\Lambda(g)$. Then so does for $f$ on $\Lambda(f)$. Absurd. Thus $f$ has no Li-Yorke pairs.

Remark 4.2. Lemma 4.1 fails if End $(X)$ is not closed (See [1], Example 2).

Lemma 4.3. Let $X$ be a dendrite with End $(X)$ countable and closed and let $f: X \rightarrow X$ be a continuous map. Suppose that $f$ has no Li-Yorke pairs. Then $f_{\mid \Lambda(f)}$ is equicontinuous at any point in $\Lambda(f) \backslash\left(P(f) \cap \operatorname{End}(X)^{\prime}\right)$.

Proof. Let $x \in \Lambda(f) \backslash\left(P(f) \cap \operatorname{End}(X)^{\prime}\right)$. Then there is a minimal set $M$ in $X$ containing $x$. We distinguish two cases. 
Case 1: $x \notin P(f)$. Then $M$ is infinite. Let $I$ be an $N$-periodic free arc in $X$ such that $M \subset \operatorname{Orb}_{f}(I)$. There is $k \geq 0$ such that $I$ is a neighborhood of $f^{k}(x)$. Since $f_{\mid I}^{N}$ has no Li-Yorke pairs, then by Theorem $1.1 f^{N}$ is equicontinuous at $f^{k}(x)$. Hence $f$ is equicontinuous at $x$.

Case 2: $x \in P(f)$. Then $x \notin E n d(X)^{\prime}$. Without loss of generality, we can assume that $f(x)=x$. As $\operatorname{End}(X)$ is closed, there is a neighborhood $V=\cup_{i=1}^{r}\left[x, e_{i}\right]$ of $x$ such that $V \cap B(X) \subset\{x\}$. Fix $\varepsilon_{0}>0$. There is $0<\varepsilon<d(x, X \backslash V)$ such that for all $0 \leq i \leq 2 r$, $d\left(f^{i}(u), f^{i}(v)\right)<\varepsilon_{0}$ whenever $d(u, v)<\varepsilon$. There is $\delta>0$ such that $d\left(x, f^{i}(y)\right)<\varepsilon$ for all $0 \leq i \leq 2 r$ whenever $d(x, y)<\delta$. Let $y \in \Lambda(f) \cap B(x, \delta)$. Then $f^{i}(y), f^{j}(y) \in\left[x, e_{s}\right)$ for some $0 \leq i<j \leq r$ and $1 \leq s \leq r$. We can assume that $i=0$ and $y \in\left(x, f^{j}(y)\right)$. If $y \notin P(f)$, as $\omega_{f}(y)$ has no isolated points, we can assume that $\left(y, f^{j}(y)\right) \subset(w, z)$ for some $w, z \in \omega_{f}(y) \cap\left[x, e_{s}\right]$. Let $Y$ be the connected component of $X \backslash\{x\}$ containing $y$.

Claim 1. $O_{f^{j}}(y) \subset Y$.

Proof. Suppose the contrary. Let $p>1$ be the smallest integer for which $f^{p j}(y) \notin Y$. Then there is $x^{\prime} \in\left(y, f^{j}(y)\right)$ such that $f^{(p-1) j}\left(x^{\prime}\right)=x$. So $\left[y, x^{\prime}\right],\left[x^{\prime}, f^{j}(y)\right]$ is an arc horseshoe, absurd. This finishes the proof of Claim 1.

Claim 2. $\left[y, f^{j}(y)\right] \cap \operatorname{Fix}\left(f^{j}\right) \neq \emptyset$.

Proof. Let $q>1$ be the smallest integer such that $f^{q j}(y) \in\left[x, f^{j}(y)\right)$. Then by Lemma 3.2 there is a point $a \in F i x\left(f^{j}\right)$ such that $b:=$ $r_{\mid\left[O r b_{f j}(y)\right]} \in\left[f^{n j}(y), f^{(n+1) j}(y)\right]$ for some $n \geq 0$. Let $b^{\prime} \in\left(y, f^{j}(y)\right) \cap$ $f^{-n j}(b)$. If $b \notin\left(y, f^{j}(y)\right)$ then $\left[y, b^{\prime}\right],\left[b^{\prime}, f^{j}(y)\right]$ is an arc horseshoe. Absurd. So $b \in\left(y, f^{j}(y)\right)$. As $\left[y, f^{j}(y)\right]$ is a free arc, $f^{j}(b)=b$. This finishes the proof of Claim 2.

Claim 3. $\operatorname{Orb}_{f^{2 j}}(y) \subset[x, b]$.

Proof. Suppose that $f^{2 j}(y) \notin[x, y]$. As $y \in R\left(f^{j}\right)$, there is $n>1$ such that $f^{j n}(y) \in(x, y)$. Suppose that $n$ is the smallest one. So there is $b^{\prime} \in\left[f^{j}(y), f^{2 j}(y)\right]$ such that $f^{(n-2) j}\left(b^{\prime}\right)=b$. Thus $\left[f^{j}(y), b^{\prime}\right],\left[b^{\prime}, f^{2 j}(y)\right]$ is an arc horseshoe. Absurd. So we get, $f^{2 j}(y) \in$ $[x, b]$. The connected subset of $[x, b] \backslash\left(\cup_{n \geq 0} f^{-n j}(b) \cup \cup_{n \geq 0} f^{-n}(x)\right)$ containing $\left[y, f^{2 j}(y)\right]$ is $f^{2 j}$-invariant. So $f^{2 n j}(y) \in[x, y]$ for all $n \geq 0$. This finishes the proof of Claim 3 .

Now, we have $d(x, y)<\delta$. So $d\left(x, f^{2 n j}(y)\right) \leq d\left(x, f^{j}(y)\right)<\varepsilon$ for all $n \geq 0$. So $d\left(x, f^{2 n j+m}(y)\right)<\varepsilon_{0}$ for any $0 \leq m \leq 2 r$ and $n \geq 0$. Thus $f$ is equicontinuous at $x$. 
Lemma 4.4. There is a dendrite $X$ with $\operatorname{End}(X)$ is countable and closed and a continuous map $f: X \rightarrow X$ without Li-Yorke pairs such that $\Lambda(f)=$ $P(f)$ but the collection of minimal sets is not closed in $2^{X}$. As a consequence, $f_{\mid \Lambda(f)}$ is not equicontinuous.

\section{Proof. Construction of the dendrite $X$.}

We construct the dendrite $X$ on the plane $\mathbb{R}^{2}$ as follows.

- Let $I_{0}=\left[e_{0}, e_{-1}\right]$ where $e_{0}=(1,0)$ and $e_{-1}=(-1,0)$,

- For any $n \geq 1$, let $J_{n}=\left[b_{n, 0}, e_{n}\right]$ where $b_{n, 0}=\left(1-\frac{1}{n}, 0\right)$ and $e_{n}=$ $\left(1-\frac{1}{n}, \frac{1}{n}\right)$

- For any $n \geq 1$ and $k \geq 1$, let $I_{n, k}=\left[b_{n, k}, e_{n, k}\right]$ where $b_{n, k}=(1-$ $\left.\frac{1}{n}, \frac{1}{n}\left(1-\frac{1}{k+1}\right)\right)$ and $e_{n, k}=\left(1-\frac{1}{n}-\frac{1}{2^{k+n}}, \frac{1}{n}\left(1-\frac{1}{k+1}\right)\right)$.

Then $X=I_{0} \cup \bigcup_{n>1} J_{n} \cup \bigcup_{n, k>1} I_{n, k}$ is a dendrite with $\operatorname{End}(X)=\left\{e_{n} ; n \geq\right.$ $-1\} \cup\left\{e_{n, k} ; n, k \geq 1\right\}$ which is countable and closed.

\section{Construction of the map $f$.}

The notation $[a, b] \rightarrow f[c, d]$ means that $f$ send linearly the arc $[a, b]$ to the arc $[c, d]$ such that $f(a)=c$ and $f(b)=d$. We define the map $f: X \rightarrow X$ as follows. For all $n, k \geq 1$,

- $f\left(e_{1}\right)=f\left(e_{0}\right)=e_{0}$,

- $\left[b_{n, 0}, b_{n+1,0}\right] \rightarrow^{f}\left[b_{n+1,0}, b_{n+2,0}\right]$,

- $\left[b_{1, k-1}, b_{1, k}\right] \rightarrow f\left[b_{k+1,0}, b_{k+2,0}\right]$,

- $\left[b_{1,0}, e_{-1}\right] \rightarrow^{f}\left[b_{2,0}, e_{2,1}\right]$ and $\left[b_{1, k}, e_{1, k}\right] \rightarrow^{f}\left[b_{k+2,0}, e_{k+2,1}\right]$,

- $\left[b_{2,0}, b_{2,1}\right] \rightarrow f\left[b_{3,0}, b_{1,0}\right]$ and $\left[b_{2, k}, b_{2, k+1}\right] \rightarrow f\left[b_{1, k-1}, b_{1, k}\right]$,

- $\left[b_{n+1,0}, b_{n+1,1}\right] \rightarrow f\left[b_{n+2,0}, b_{n, 0}\right],\left[b_{n+1,1}, b_{n+1,2}\right] \rightarrow f\left[b_{n, 0}, b_{n, 2}\right]$ and $\left[b_{n+1, k-1}, b_{n+1, k}\right] \rightarrow^{f}$ $\left[b_{n, k}, b_{n, k+1}\right]$

- $\left[b_{2,1}, e_{2,1}\right] \rightarrow^{f}\left[b_{1,0}, e_{-1}\right]$ and $\left[b_{n+1, k}, e_{n+1, k}\right] \rightarrow^{f}\left[b_{n, k+1}, e_{n, k+1}\right]$.

It is easy to check that the map $f: X \rightarrow X$ is continuous, has no LiYorke pairs and the collection of minimal sets is not closed in $2^{X}$. We have $\Lambda(f)=P(f)=\left\{e_{0}, e_{-1}\right\} \cup\left\{e_{n, k} ; n, k \geq 1\right\}$. Indeed, for any $n \geq 1, \operatorname{Orb}_{f}\left(e_{n, 1}\right)$ is a periodic orbit which converges in $2^{X}$ to the set $M=\left\{e_{n} ; n \geq 1\right\}$. Since $f\left(e_{0}\right)=e_{0}$, then $M$ is not minimal. (See Figures 1 and 2) 


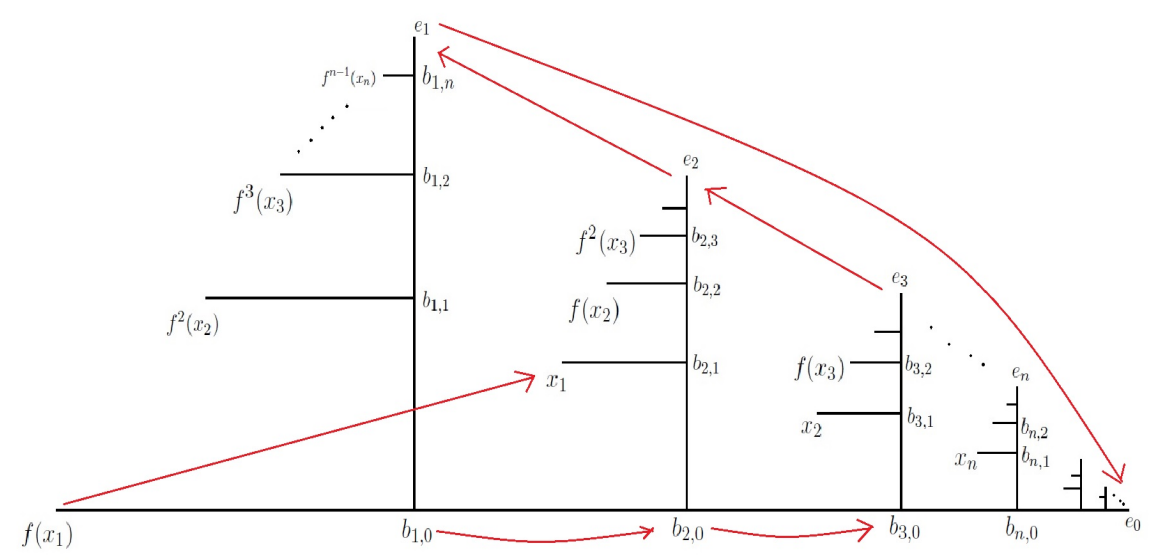

FIGURE 1 . The action of $f$ on $I_{0}$ and $\left\{e_{n} ; n \geq-1\right\}$

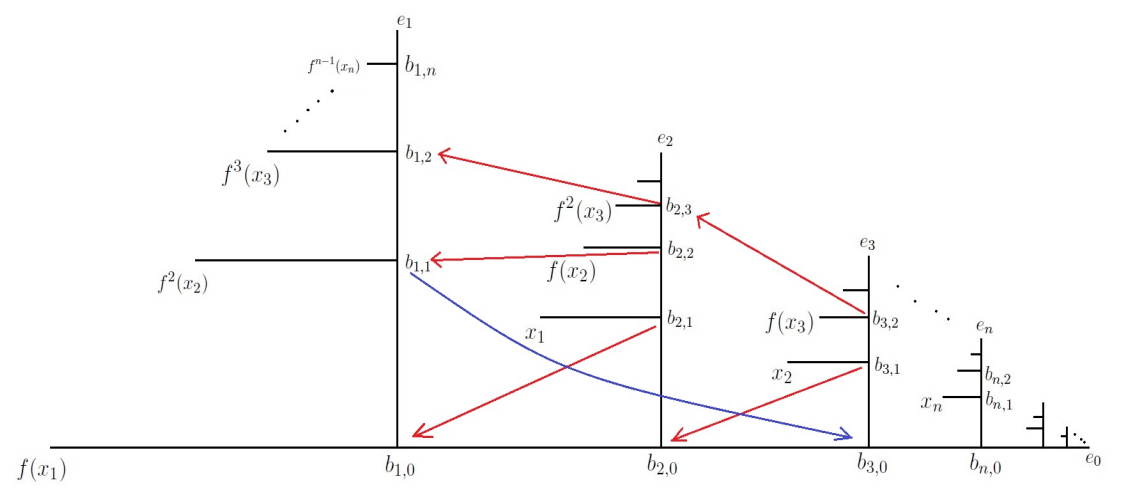

FIgURE 2. The action of $f$ on branch points $b_{n, k}(n \geq 1, k \geq 0)$

Lemma 4.5. Let $X$ be a dendrite with End $(X)$ countable and closed and let $f: X \rightarrow X$ be a continuous map. If $f$ has no Li-Yorke pairs then $R(f)=R R(f)$.

Proof. It suffices to prove that $R(f) \subset R R(f)$. Let $x \in R(f)$. Then $M=$ $\omega_{f}(x)$ is a minimal set. If $x \in P(f)$ then $x \in R R(f)$. Suppose that $x \notin P(f)$. Then there is a $p$-periodic free arc $I$ in $X$ such that $M \subset \operatorname{Orb}_{f}(I)$. Let $0 \leq i<p$ such that $x \in f^{i}(I)$. There is $x^{\prime} \in I \cap M$ such that $f^{i}\left(x^{\prime}\right)=x$. Let $U$ be an open set in $X$ containing $x$. Then $f^{-i}(U)$ is an open subset in $X$ containing $x^{\prime}$. By Theorem $1.1 R\left(f_{\mid I}^{p}\right)=R R\left(f_{\mid I}^{p}\right)$. As $x^{\prime} \in \omega_{f_{\mid I}^{p}}\left(x^{\prime}\right)$, we get 
$x^{\prime} \in R R\left(f_{\mid I}^{p}\right)$. So there is an integer $N>0$ such that $\operatorname{Orb}_{f^{p N}}\left(x^{\prime}\right) \subset f^{-i}(U)$. It follows that $\operatorname{Orb}_{f^{p N}}(x) \subset U$. Thus $x \in R R(f)$. We conclude that $R(f)=$ $R R(f)$.

In the following Lemma, we strengthen absence of Li-Yorke pairs to get equicontinuity on $\Lambda(f)$.

Lemma 4.6. Let $X$ be a dendrite with End $(X)$ countable and closed and let $f: X \rightarrow X$ be a continuous map. Suppose that $f$ has no Li-Yorke pairs. If one of the following assertions holds

(1) the collection of minimal sets is closed in $\left(2^{X}, d_{H}\right)$,

(2) $f_{\mid \Lambda(f)}$ is equicontinuous at every point in $\operatorname{End}(X)^{\prime}$,

(3) $f_{\mid P(f)}$ is equicontinuous,

then $f_{\mid \Lambda(f)}$ is equicontinuous.

Proof. (1) Suppose that the collection of minimal sets is closed in $\left(2^{X}, d_{H}\right)$. Let $x \in \Lambda(f)$. By Lemma 4.3 we can assume that $x \in P(f)$. Also we may assume that $x \in \operatorname{Fix}(f)$. Let $\left(y_{n}\right)_{n \geq 1}$ be a sequence in $\Lambda(f)$ converging to $x$. For any $n \geq 1, M_{n}=\omega_{f}\left(y_{n}\right)$ is a minimal set containing $y_{n}$. We can assume that $\left(M_{n}\right)$ is convergent to some closed set $M$. Then $d\left(y_{n}, M\right)$ goes to 0 when $n \rightarrow+\infty$. As the collection of minimal sets is closed in $\left(2^{X}, d_{H}\right)$, then $M$ is a minimal set. So $M=\{x\}$. Now fix $\varepsilon>0$. There is $\alpha>0$ such that if $d(x, y)<\alpha$ then $d\left(x, \omega_{f}(y)\right)<\varepsilon$. This implies that $d\left(f^{n}(x), f^{n}(y)\right)<\varepsilon$ for all $n \geq 0$. thus $f$ is equicontinuous at $x$.

(2) and (3) follows from Lemma 4.3

Proof of the main Theorem The implications $(2) \Rightarrow(1),(2) \Rightarrow(4)$ and $(1) \Rightarrow(3)$ are given in Lemmas 4.1, 2.8 and 4.5. The implication $(1) \Rightarrow(5)$ is proved in Lemma 2.7 . Finally, $(1) \nRightarrow(4)$ is given by Lemma 4.4.

\section{REFERENCES}

1. G. Askri, Li-Yorke chaos for dendrite maps with zero topological entropy and $\omega$-limit sets, Discrete and Continuous Dynamical Systems, 37(6), (2017), 2957-2976.

2. L. S. Block and W. A. Coppel, Dynamics in One Dimension, Lecture Notes in Math., vol. 1513, Springer-Verlag, 1992.

3. F. Balibrea, J. L. G. Guirao and M. Lampart, A note on the Definition of $\alpha$-limit Set, Appl. Math. Inf. Sci. 7, No. 5, (2013), 1929-1932.

4. F. Blanchard, E. Glasner, S. Kolyada and A. Maass, On Li-Yorke pairs, J. Reine Angew. Math. 547, (2002), 51-68.

5. V. V. Fedorenko, S. F. Kolyada, A. N. Sarkovskii and A. G. Sivak, Dynamics of one dimensional mapping, Naukova Dumka, Kiev, (1989). (Russian).

6. V. V. Fedorenko, A. N. Sarkovskii and J. Smital, Characterizations of Weakly Chaotic Maps of the Interval, Proceedings of the American Mathematical Society 110(1), (1990), 141-141.

7. Z. Kocan, V. K. Kurkova and M. Malek, Entropy, horseshoes and homoclinic trajectories on trees, graphs and dendrites, Ergod. Th. Dynam. Sys, 31, (2011), 165-175.

8. S. Kolyada, L. Snoha and S. Trofimchuk, Noninvertible minimal maps, Fund. Math, 168, (2001), 141-163. 
9. D. Preiss and J. Smital, A characterisation of non-chaotic maps of the interval stable under small perturbations, Trans. AMS, 313, N. 2, (1989), 687-696.

10. H. Marzougui and I. Naghmouchi, On totally periodic $\omega$-limit sets, Houston journal of mathematics, 43(4) (2017),1291-1303.

11. J. Mai and E. Shi, $\bar{R}=\bar{P}$ for maps of dendrites $X$ with $\operatorname{Card}(\operatorname{End}(X))<c$, Int. Journ. Bif. Chaos, 19, No. 4, (2009), 1391-1396.

Ghassen Askri, Bizerte Preparatory Engineering Institute, 7021, Jarzouna, TUNISIA

E-mail address: askri.ghassen@gmail.com and elaskri.ghassen@ipeib.u-carthage.tn 